\title{
A Study on Control Strategy under Tip-in Condition Based on AMT Vehicle
}

\author{
Gao Yang ${ }^{a}$, Chen Yong ${ }^{b}$, Luo Daguo ${ }^{c}$, Liu Wenzhong ${ }^{d}$, Gu Mingqie and Zhao \\ Fuquan $^{f}$
}

3366 Wei Wu Road, Linjiang Industrial Park, Xiaoshan District of Hangzhou, Zhejiang Province, China

aemail: auto_gaoyang@163.com, bemail: Cheny02@geely.com, 'email: luodaguo@rd.geely.com, demail: liuwenzhong@rd.geely.com, eemail: gumingqi@126.com, 'email: zhaofuquan10@163.com

\section{Keywords: AMT, Hard Acceleration, Control Strategy, Simulation Platform}

\begin{abstract}
The control strategy of gear-position optimization of an AMT vehicle under Tip-in condition is presented in this article. The down-shift principle of an automatic transmission under an acceleration condition is also analyzed. The in-the-loop simulation platform of transmission system software of an AMT vehicle is established using the Matlab/Simulink. The simulation platform mainly includes the vehicle load module and the control strategy module. The vehicle load module mainly includes the driver intention module, the transmission model, the engine model, the synchronizer model, the clutch model and the resistance model. The hard-acceleration condition is simulated under no deceleration condition by utilizing the optimized control strategy and conventional control strategy respectively. The result indicates that the proposed control strategy can identify a driver's intention, decrease shift times, reduce power interruption time, improve comfort and make the vehicle reach the maximum speed quickly.
\end{abstract}

\section{Preface}

The tradition AMT shift schedule mainly determines the target gear according to the throttle and the speed, which can make the vehicle work better power and economy conditions. It will increase the throttle opening suddenly when the vehicle accelerating. In this case, to make the engine work in reasonable conditions, the transmission gear will be reduced. If you do not impose restrictions, It will bring the state that it even just downshifts and then upshifts. Which does not meet the intent of the driver, and also reduces the power, the economy, the comfort and the transmission life. According to analyzing the transmission downshift principle in the Tip-in conditions, determined whether the downshift is reasonable, it presents automated mechanical transmission(AMT) vehicles gear optimal control strategy in the Tip-in condition. Built AMT vehicle drive system software in-the-loop simulation platform with Matlab / Simulink software. Simulate with the optimal control strategy in-the-loop simulation platform, The simulation results verify the correctness and the superiority of the proposed control strategy.

\section{Downshift principle in the accelerating conditions}

Now, the main application of the automatic transmission is a two-parameter shift schedule determined by the throttle opening and the vehicle speed. The principle of the shift schedule is shown in Figure $1 . \mathrm{m}+1 \rightarrow \mathrm{m}$ curve is a downshift law curve; $\mathrm{m} \rightarrow \mathrm{m}+1$ curve is a upshift law curve. In the A to $\mathrm{B}$ stage the transmission is in the $\mathrm{m}$ gear and the throttle keeps $\mathrm{P}_{\text {low }}$ unchanged. The transmission will upshift to $\mathrm{m}+1$ gear when the speed increases to the point $\mathrm{B}$, because it crosses the current upshift curve. The transmission is in the $\mathrm{m}+1$ gear in the $\mathrm{B}$ to $\mathrm{C}$ stage, the throttle keeps $\mathrm{P}_{\text {low }}$ constant continually, the speed continues to increase, until the point $\mathrm{C}$. The $\mathrm{C}$ to $\mathrm{E}$ stage is the process that the throttle increases from the $\mathrm{P}_{\text {low }}$ to the $\mathrm{P}_{\text {high }}$ suddenly. At this time the speed does not change due to the vehicle inertia. But this process crosses the point $\mathrm{D}$ that is the intersection of the current state of the law of a downshift curve and $\mathrm{CE}$ line, which makes the transmission reduce from $\mathrm{m}+1$ gear to $\mathrm{m}$ gear. 
The transmission is in the $\mathrm{m}$ gear in the $\mathrm{E}$ to $\mathrm{F}$ stage. The throttle keeps $\mathrm{P}_{\text {high }}$ constant with the speed increasing until it get to the point $\mathrm{F}$. The transmission will upshift to $\mathrm{m}+1$ gear because it crosses the current upshift curve. The transmission is in the $\mathrm{m}+1$ gear in the $\mathrm{F}$ to $\mathrm{G}$ stage. The throttle continually keeps $\mathrm{P}_{\text {high }}$ constant with the speed increasing. In the urgent reducing throttle process the transmission will keeps the vehicle working in the corresponding mode based on this shift schedule, so it will be frequently shifting action.

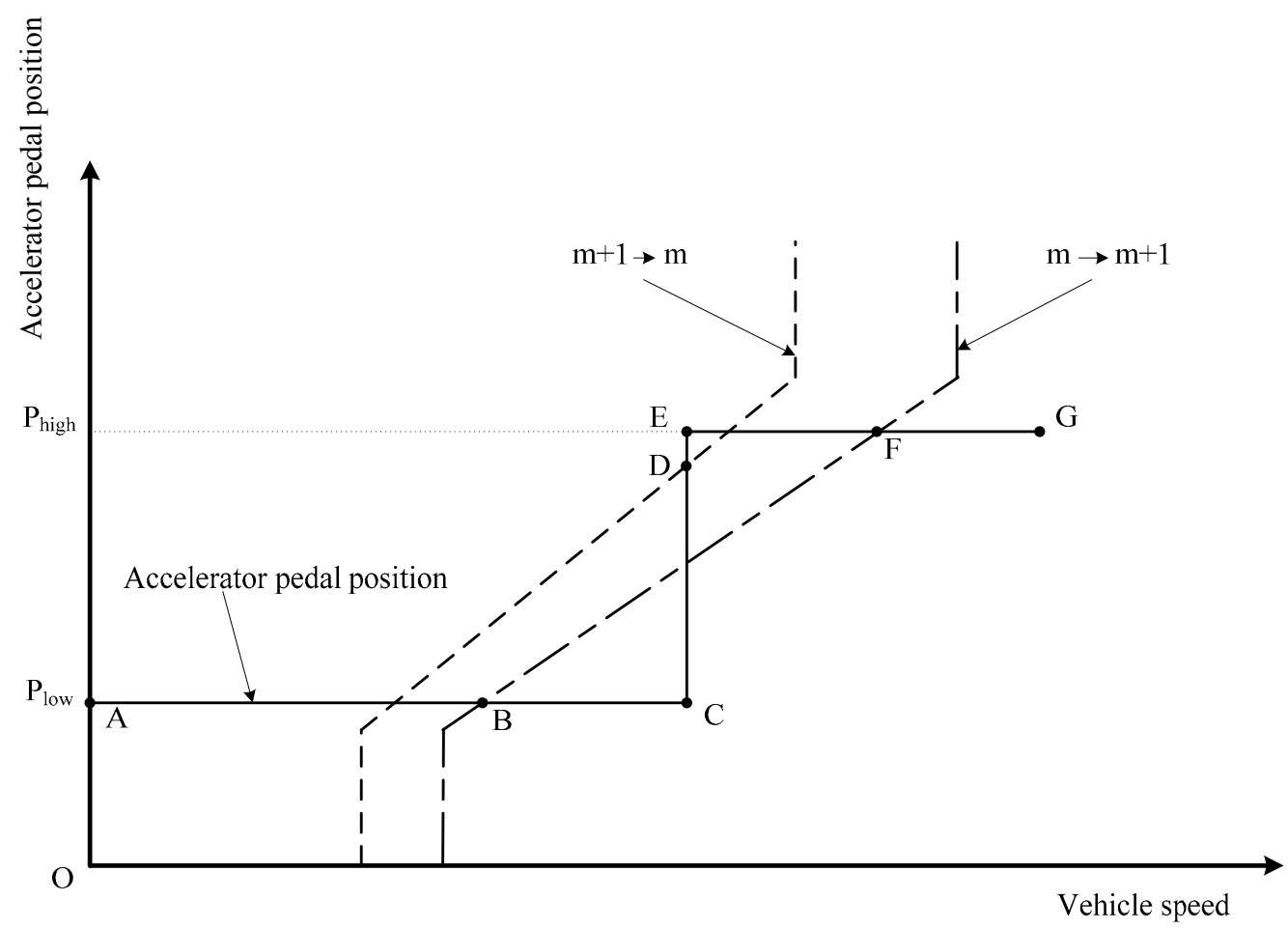

Fig. 1 the downshift principle in the urgent reducing throttle conditions

\section{Optimal control strategy}

Engine operating conditions under different speed, the throttle and the gear can be gained according to the engine model. To measure the time shift in different shift strategy, establish the following speed balance equation.

$$
v_{1}=\left\{\begin{array}{cc}
v_{0}+a_{1}\left(t_{\text {downshift }}-t_{-1}-n t_{+1}\right) & ; \text { downshift } \\
v_{0}+a_{0} t_{\text {notdownshift }} & ; \text { not downshift }
\end{array}\right.
$$

In this equation: $V_{1}$ is downshift and then rise to the speed of the original gear; $V_{0}$ is the speed begining to accelerate; $a_{0}$ is acceleration under the original gear after accelerating; $a_{1}$ is acceleration under the new gear after accelerating; $t_{\text {notdownshift }}$ is time to accelerate to V1 under the original gear after accelerating; $t_{\text {downshift }}$ is time to accelerate to V1 under the new gear after accelerating; $n$ is actual downshift number; $t_{-1}$ is average downshift time; $t_{+1}$ is average upshift time.

Build the process shown in Figure 2 of the optimize shift control strategy in the Tip-in condition. $m$ is the intermediate variables in the program. In some conditions the engine is not working properly until the transmission shifts, so it make whether the engine still be able to work properly as the judging condition in the current gear. It make $t_{\text {downshift }} \geq t_{\text {notdownshift }}$ as a judge for the comparison of the two shift strategies power. 


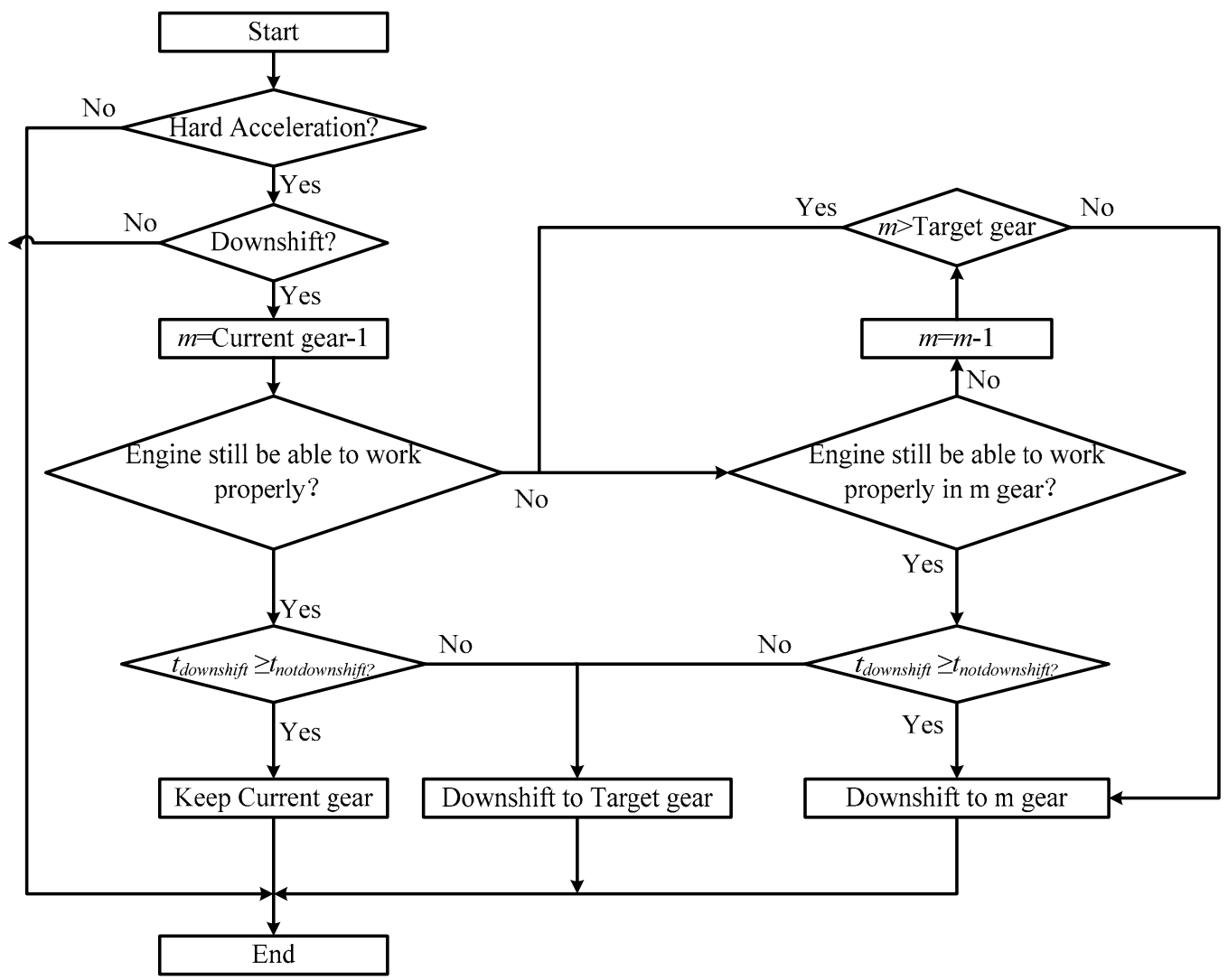

Fig.2 Flow of the optimize shift control strategy

\section{Software-in-the-loop simulation platform}

Built an automatic transmission vehicle drive system software in-the-loop simulation platform with Matlab / Simulink software. It shown in Figure $3^{[1-3]}$.

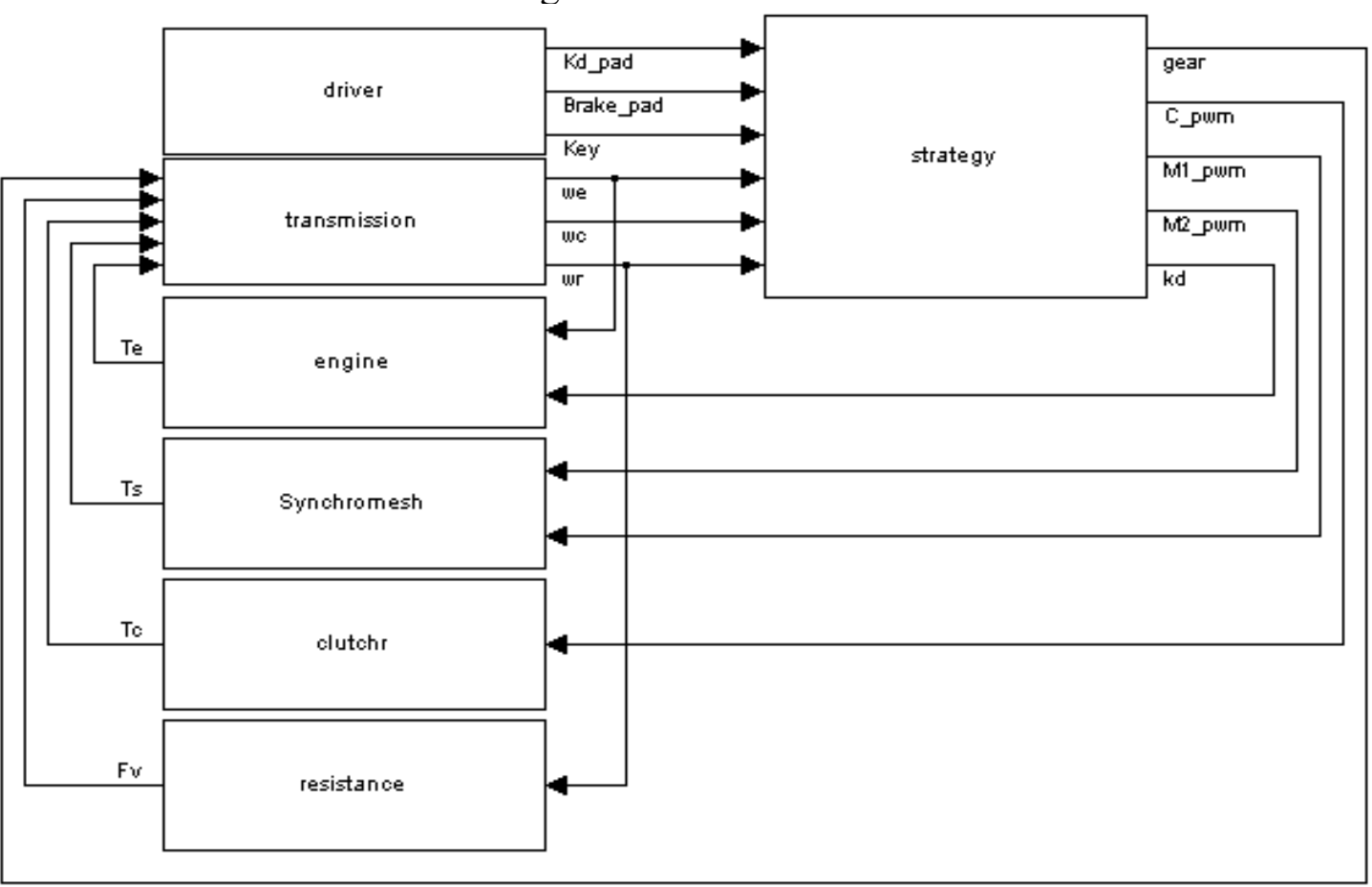

Fig.3 Simulation platform interface

The simulation platform mainly includes the driver intention module, the transmission model, the engine model, the synchronizer model, the clutch model, the resistance model and the control strategy 
module ${ }^{[5-6]}$. $\mathrm{kd}$ is the actual throttle opening; we is engine speed; wc is input shaft speed; C_pwm is the control signal of the clutch actuator motor; M1_pwm is the control signals of the election and shift implementation motor 1; M2_pwmv is the control signals of the election and shift implementation motor 2; gear is the gear the transmission needs; Te is the engine output torque; Tc is the torque which the clutch can be passed; Ts is the torque which the synchronizer can be passed; Fv is the vehicle resistance; $\mathrm{wr}$ is the tire angular velocity; $\mathrm{Kd}$ pad is the accelerator pedal position; Brake_pad is the brake pedal position; Key is the ignition switch.

\section{Simulation analysis}

A equipped with automatic mechanical transmission, electronically controlled engines and the dry clutch vehicle as the study object. Simulate and analyse to the result that the vehicle is controlled using the optimal control strategy and the traditional control strategy respectively on the built drive system software in-the-loop simulation platform ${ }^{[4-5]}$. The Simulation conditions: The initial accelerator pedal position is $20 \%$; The initial speed is $0.7 \mathrm{~km} / \mathrm{h}$; The first gear transmission ratio is 6.194 ; The second gear transmission ratio is 3.894; The third gear transmission ratio is 2.260; The fourth gear transmission ratio is 1.428; The fifth gear transmission ratio 1.000; The transmission initial gear is empty; The rolling resistance coefficient is 0.02 ; The driving road ramp angle is 0rad; The wind speed to the face is $0 \mathrm{~km} / \mathrm{h}$; The clutch has completely separated before the simulation starting. The tradition two-parameter shift schedule of the automatic transmission based on the throttle and the speed is shown in Figure 4.

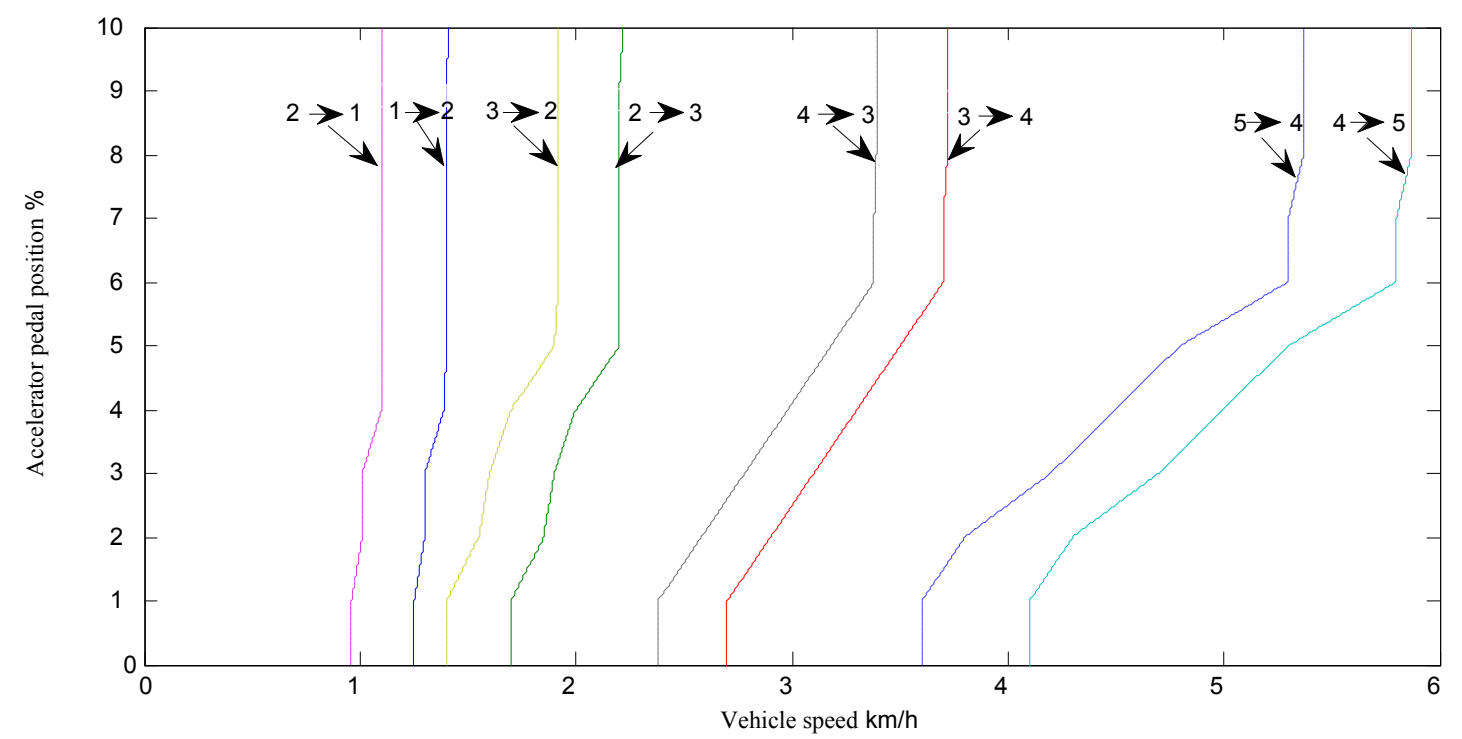

Fig.4 The curve of the two-parameter shift schedule

The figure 5 is the simulation curve that the throttle opening, the speed and the gear of the traditional shift schedule changes along with the acceleration time. The figure 6 is the simulation curve that the throttle opening, the speed and the gear of the optimize shift schedule changes along with the acceleration time. From the figure 5 it can be seen that the speed increasing the throttle suddenly is $30 \mathrm{~km} / \mathrm{h}$ and the transmission has been promoted to the fourth gear before the throttle increases. The increased throttle opening is $70 \%$. The transmission drops to the third gear from the fourth gear and then rising to the fourth gear and the fifth gear. After shifting three times it reaches the maximum speed which uses 80 s. From the figure 6 it can be seen that the speed increasing the throttle suddenly is also $30 \mathrm{~km} / \mathrm{h}$ and the transmission has been promoted to the fourth gear before the throttle increases. The increased throttle opening is $70 \%$. The transmission rises from the fifth gear to the fourth gear only one time. It reaches the maximum speed which uses $77 \mathrm{~s}$. 

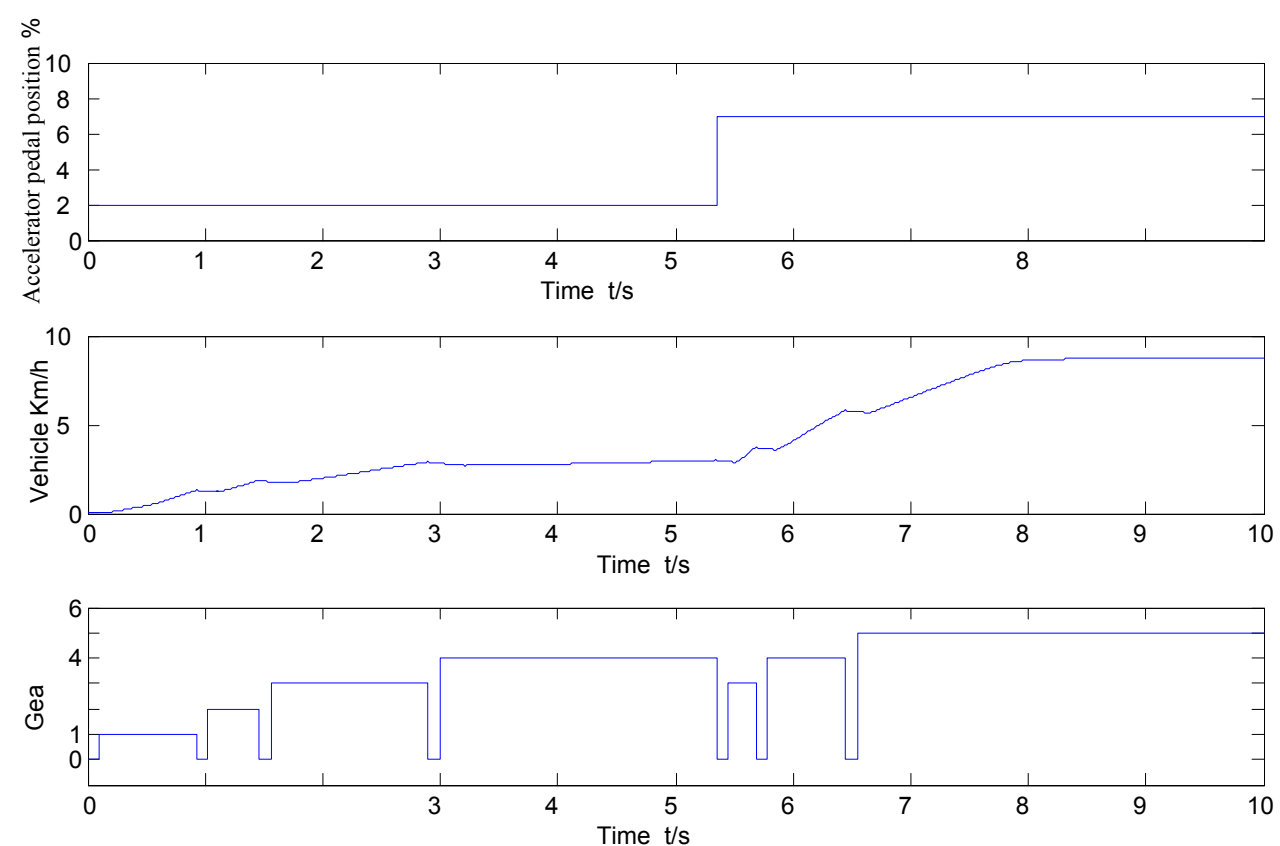

Fig.5 The simulation curve of the traditional control strategy
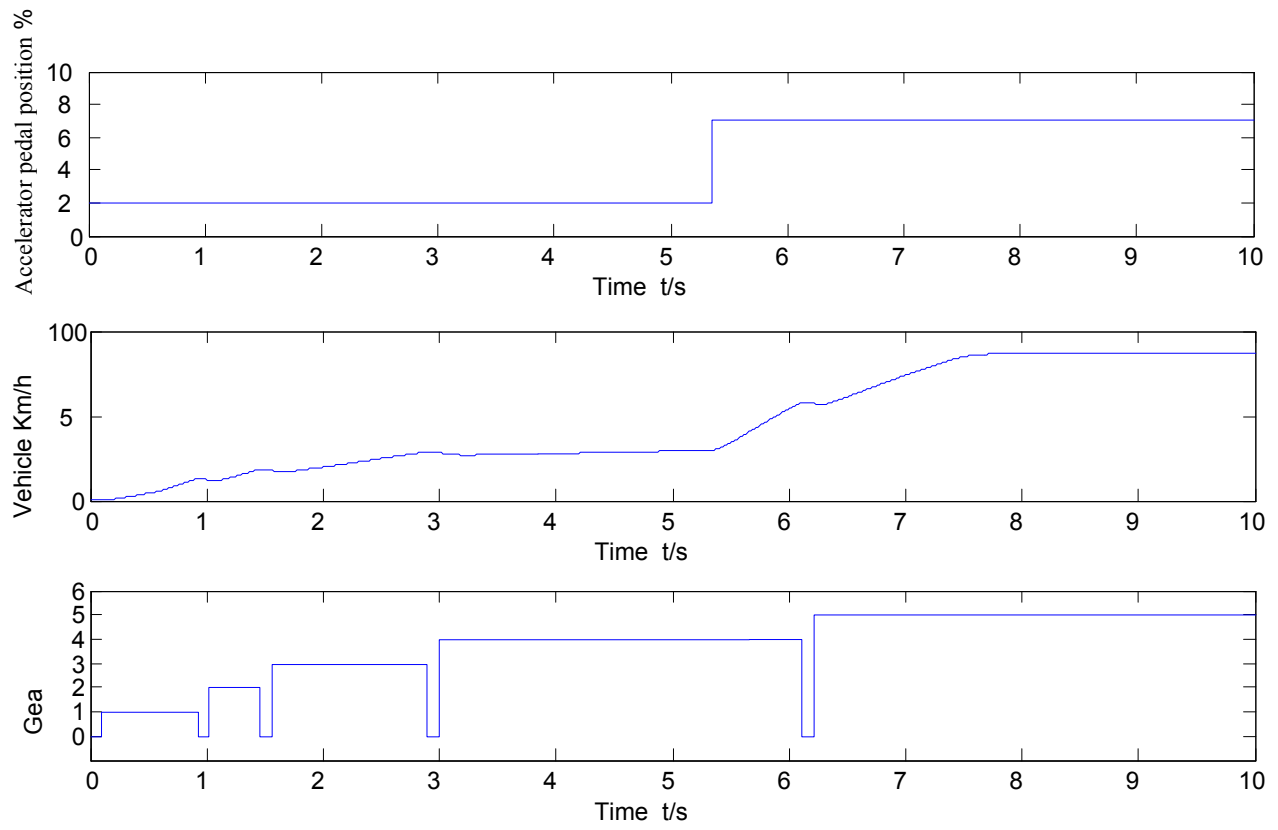

Fig.6 The simulation curve of the optimize control strategy

The simulation results showed that: After increasing the throttle in the same condition, the proposed optimal control strategy could identify the driver intention, reduced shift times and the power break time, improved comfort ability, and reduced the time that the vehicle achieved maximum speed.

\section{References}

[1] Zhang Tai, Ge Anlin, Tang Chunxue, et al. A Study on Shift Schedule of Off-road Vehicle with TC and AMT, J. Automotive Engineering, 2007, 29(3): 226-229,249 (In Chinese). 
[2] Guo Xiang en1, Li Yan. Study on Dynamic Shift Schedule of Construction Vehicle, J. Journal of Wuhan University of Technology(Transportation Science \& Engineering), 2009, 33(5): 839-842(In Chinese).

[3] DONG Yue-hang, YIN Cheng-liang, CHEN Li, et al. Optimal Torque Control Strategy of Electric Transmission Driver Based on AMT During Gear Shift, J. Journal of Shanghai Jiaotong University, 2009, 43(2): 217-221(In Chinese).

[4] Yuhai Wang, Jian Song and Xingkun Li. Simulation of AMT Autoshift Process Based on Matlab/Simulink/Stateflow, C. //SAE Paper 2004-01-2055.

[5] Xiaofeng Yin, Jingxing Tan, Yulong Lei, et al. Combined Control Strategy for Engine Rotate Speed in the Shift Process of Automated Mechanical Transmission, C. // SAE Paper 2004-01-0427. 